\title{
Accounting for effective interactions among charged microgels
}

\author{
L. A. Aguirre-Manzo, ${ }^{1}$ M. Ledesma-Motolinía,${ }^{2}$ L. F. Rojas-Ochoa, ${ }^{1}$ V. Trappe,${ }^{3}$ \\ J. Callejas-Fernández, ${ }^{4}$ C. Haro-Pérez, ${ }^{2, *}$ and P. González-Mozuelos ${ }^{1, \dagger}$ \\ ${ }^{1}$ Departamento de Física, Cinvestav del I. P. N., Av. Instituto Politécnico Nacional 2508, 07360 Ciudad de México, Mexico \\ ${ }^{2}$ Departamento de Ciencias Básicas, Universidad Autónoma Metropolitana-Azcapotzalco, 02200 Ciudad de México, Mexico \\ ${ }^{3}$ Departement de Physique, Université de Fribourg, 1700 Fribourg, Switzerland \\ ${ }^{4}$ Departamento de Física Aplicada, Universidad de Granada, 18071 Granada, Spain
}

(Received 21 June 2019; published 3 September 2019)

\begin{abstract}
We introduce a theoretical approach to describe structural correlations among charged permeable spheres at finite particle concentrations. This theory explicitly accounts for correlations among microions and between microions and macroions and allows for the proposal of an effective interaction among macroions that successfully captures structural correlations observed in poly- $N$-isopropyl acrylamide microgel systems. In our description the bare charge is fixed and independent of the microgel size, the microgel concentration, and the ionic strength, which contrasts with results obtained using linear response approximations, where the bare charge needs to be adapted to properly account for microgel correlations obtained at different conditions.
\end{abstract}

DOI: 10.1103/PhysRevE.100.032602

\section{INTRODUCTION}

The structural and thermodynamic properties of suspensions of charged colloidal particles are regulated by the electrostatic interactions among their ionic components [1]. For nonpermeable colloidal particles, the macroion-macroion correlations are generally well described by the DerjaguinLandau-Verwey-Overbeek potential, which presents a combination of short-ranged interactions, including a hard-sphere repulsion and a van der Waals attraction and a long-ranged screened Coulomb (Yukawa) potential [1,2].

However, modeling the interactions among permeable charged spheres, such as ionic microgels, is less obvious. Microgels are mesoscopic spherical particles formed by crosslinked polymers that swell in the suspending solvent, the degree of swelling being controlled by $\mathrm{pH}$, hydrostatic pressure, ionic strength, and/or temperature [3-10]. Expansion and contraction of microgels should in principle not affect the bare charge of the microgels, but the effective interactions among them will depend on the internal charge distribution, which in turn is a function of the degree of swelling [11-15]. This is related to the free flow of the mobile microions in and out of the volume of the microgel, a process that is controlled by electrostatic and excluded volume interactions [13].

Previous experimental results obtained for poly- $N$ isopropyl acrylamide (PNiPAM) microgels at dilute, quasideionized conditions were modeled with the PoissonBoltzmann cell model (PBCM), allowing for the conclusion that the number of counterions entrapped within the microgels increases on shrinking; this results in a reduction of

\footnotetext{
*cehp@azc.uam.mx

†pedro@ fis.cinvestav.mx
}

the microgel net internal charge, i.e., bare charge minus the number of salt ions within the microgel and, consequently, in a reduction of structural correlations among the microgels (macroions) [4]. The PBCM computations, however, neglect not only the short-ranged interactions between the polymer segments and the microions but also the nonlinear correlations among microions, such that an extension to the description of structural correlations in systems with larger microgel concentrations or larger ionic strength is rather unclear.

In this work we propose a different approach for modeling structural correlations among microgels. We consider the microgels as permeable charged spheres and the microions as small charged hard spheres. By applying the dressed ion theory (DIT) to the effective potential between macroions we deconvolute the short- and long-range interactions [14]. DIT connects the short-ranged components of the direct correlation functions to the long-ranged behavior of the effective interactions among the particles belonging to the observable species [16]. This allows us to account for nonlinear effects, including condensation of counterions.

The strength of our theoretical approach is established by its capacity to describe the experimental structure factors of charged PNiPAM microgel systems at different conditions. For a fixed bare charge, the effective charge and screening length are results of the calculations.

\section{EXPERIMENTS}

\section{A. Sample preparation}

Our PNiPAM microgels are synthesized by using the standard dispersion polymerization described by Senff and Richtering [5]. Two solutions are prepared: the first contains $7.9 \mathrm{~g} N$-isopropylacrylamide (monomer), $0.15 \mathrm{~g}$ $N, N$-methylenbisacrylamide (crosslinker), $0.15 \mathrm{~g}$ sodium dodecylsulfate (stabilizer), and $450 \mathrm{ml}$ Milli-Q water, and the 
second contains $0.1 \mathrm{~g}$ potassium peroxodisulfate (initiator) and $50 \mathrm{ml}$ Milli-Q water. Both solutions are flushed with nitrogen for $30 \mathrm{~min}$ prior to use. After heating the first solution to $70^{\circ} \mathrm{C}$, the second solution is added, and polymerization is allowed to proceed for $5 \mathrm{~h}$ under a constant stream of nitrogen and gentle stirring, using a magnetic bar. The sample is then left to cool down to ambient temperature and filtered through glass wool. The resulting stock is further purified by dialysis and subsequently by 10 centrifuging cycles of $20 \mathrm{~h}$ duration at $11,000 \mathrm{rpm}$, where we replace after each cycle the supernatant with pure Milli-Q water. To produce systems at different ionic strength we pursue the following routes. We produce quasideionized samples by putting the microgel dispersion in contact with ionic exchangers (Amberlite IRN$150)$ in sealed quartz cells. The number concentration of the final stock dispersion is estimated from the Bragg peak positions that appears in crystalline samples [4]. For these systems, we consider the ionic strength to correspond to that expected for pure water at room temperature: $\theta_{s}=10^{-7} \mathrm{M}$. For higher ionic strength we use samples that remain in contact with the atmosphere, adding $\mathrm{NaCl}$ so that the samples contain $0 \mathrm{M}, 10^{-6} \mathrm{M}$, and $10^{-5} \mathrm{M} \mathrm{NaCl}$. To account for the contamination by $\mathrm{CO}_{2}$ for these systems we assume that the $\mathrm{CO}_{2}$ contribution corresponds to the addition of $3 \times 10^{-6} \mathrm{M}$ of monovalent electrolyte [17], such that the ionic strength of the series corresponds to $\theta_{s}=10^{-7}, 3 \times 10^{-6} \mathrm{M}, 4 \times 10^{-6} \mathrm{M}$, and $1.3 \times 10^{-5} \mathrm{M}$.

\section{B. Microgel characteristics}

As denoted in numerous previous studies, PNiPAM microgels undergo a volume phase transition at a volume phase transition temperature (VPTT) of approximately $33^{\circ} \mathrm{C}$. Below the VPTT the microgels are swollen, while they collapse to a more compact configuration above the VPTT. PNiPAM is an uncharged polymer. However, PNiPAM microgels are generally produced by using a charged initiator, like in our case the potassium peroxodisulfate. The charged initiator remains attached to dangling ends, such that the microgels acquire charge, the charged groups being primarily located within the outer layer of dangling ends of the microgels [4,18].

The temperature-dependent size of our PNiPAM microgels is determined by dynamic and static light scattering, where we use highly diluted microgel suspensions, setting the ionic strength to the conditions denoted in the previous section. To fit the data obtained in static light scattering we use a form factor that is based on Rayleigh-Gans-Debye theory and assumes a nonhomogeneous distribution of polymer segments within the microgels that are considered to be monodisperse in size [19]. The radii obtained from these fits are found to agree with the hydrodynamic radii obtained from the dynamic light-scattering data. Within the range of ionic strength investigated we do not find any noticeable dependence of the microgel size on ionic strength. A more detailed account of the dependence of the microgel radius $R_{M}$ on temperature and salt concentration is left for a future report; here we center our attention on the following conditions: $R_{M}=117 \pm 3 \mathrm{~nm}$ at $24.7^{\circ} \mathrm{C}, R_{M}=89 \pm 3 \mathrm{~nm}$ at $31.1^{\circ} \mathrm{C}, R_{M}=55 \pm 2 \mathrm{~nm}$ at $33.2^{\circ} \mathrm{C}$, and $R_{M}=51 \pm 2 \mathrm{~nm}$ at $34.9^{\circ} \mathrm{C}$.

\section{Light-scattering experiments}

All light-scattering experiments are performed with a 3D LS spectrometer device (LS Instruments AG, Fribourg, Switzerland), equipped with a He-Ne laser operating at a wavelength of $\lambda=632.8 \mathrm{~nm}$ and a multitau digital correlator (Flex). As described in previous works [20,21], this device enables us to determine the single-scattering contributions of moderately turbid samples in dynamic and static light-scattering experiments. To obtain ensemble-averaged values for the nonergodic systems, such as those forming crystals, we slowly rotate the samples during acquisition. The static structure factors $S(q)$ are then determined by dividing the ensemble averaged, scattered light intensity of the samples of interest by the form factors of the microgels [22].

\section{THEORY}

As a model for the experimental PNiPAM system, we consider a fluid containing three ionic components: microgels labeled $M$, small anions $\left(\mathrm{Cl}^{-}, \mathrm{OH}^{-}, \mathrm{HCO}_{3}^{-}\right)$labeled $a$, and small cations $\left(\mathrm{Na}^{+}, \mathrm{H}^{+}\right)$labeled $c$. We denote the number density and electric charge of species $\alpha$ by, respectively, $\rho_{\alpha}$ and $Q_{\alpha}$. All charges are indicated in elementary charge units, so that $Q_{a}=-1$ and $Q_{c}=1$, whereas the bare charge of the microgels in our experiments, $Q_{M}<0$, is an unknown quantity that we will attempt to determine by the theory described here. Obviously, the system needs to be globally electroneutral, $\rho_{M} Q_{M}+\rho_{a} Q_{a}+\rho_{c} Q_{c}=0$. The net interactions among the components are assumed to be given by a superposition of hard-sphere and electrostatic potentials, where $\sigma_{\alpha \gamma}$ is the nonadditive hard-core diameter between species $\alpha$ and $\gamma$ [i.e., not necessarily $\sigma_{\alpha \gamma}=\left(\sigma_{\alpha \alpha}+\sigma_{\gamma \gamma}\right) / 2$ ], and the $\alpha$ component has an spherical charge distribution $z_{\alpha}(r)$ such that $\tilde{z}_{\alpha}(q=0)=Q_{\alpha}$, where $\tilde{f}(q)$ denotes the Fourier transform of a function $f(r)$. For simplicity, we assume a constant charge density for $z_{\alpha}(r)$ within the interval $S_{\alpha} \leqslant r \leqslant R_{\alpha}$, and zero charge outside this interval [14]; $R_{\alpha}$ and $S_{\alpha}$ are, respectively, the external radius and internal radius of the spherical shell over which the charge of species $\alpha$ is uniformly distributed.

The small ions are assumed to be characterized by the values $\sigma_{a a}=\sigma_{a c}=\sigma_{c c}=0.6 \mathrm{~nm}, R_{a}=R_{c}=0.3 \mathrm{~nm}$, and $S_{a}=S_{c}=0.0 \mathrm{~nm}$; the small ions are thus solid charged spheres with a charge fully contained within their respective hard cores. By contrast, we assume for the permeable macroions that $\sigma_{\mathrm{Ma}}=\sigma_{\mathrm{Mc}}=0.0 \mathrm{~nm}$ and $S_{M}=0.9 R_{M}$, taking the distribution of the charge at the rim of the microgels into account.

The Fourier transform of the electrostatic contributions to the particle-particle interaction is given by $\beta \tilde{u}_{\alpha \gamma}^{\mathrm{el}}(q)=$ $\tilde{z}_{\alpha}(q) \tilde{v}(q) \tilde{z}_{\gamma}(q)$ with $\tilde{v}(q)=4 \pi l_{b} / q^{2}$, corresponding to an electrostatic potential in real space of $v(r)=l_{b} / r ; \beta \equiv$ $\left(k_{B} T\right)^{-1}$ with $k_{B}$ the Boltzmann constant and $T$ the absolute temperature of the system; $l_{b} \equiv \beta e_{p}^{2} /\left(4 \pi \varepsilon_{w} \varepsilon_{0}\right)$ is the Bjerrum length; $e_{p}$ is the elementary charge; $\varepsilon_{w}$ is the dielectric constant of water; and $\varepsilon_{0}$ is the vacuum permittivity. Within the temperature range considered experimentally, the Bjerrum length in water is considered to be constant, $l_{b}=0.71432 \mathrm{~nm}$, 
as the temperature dependence of $\varepsilon_{w}$ is essentially compensated by $1 / T$ [23].

The direct correlation function takes the form $c_{\alpha \gamma}(r)=$ $c_{\alpha \gamma}^{s}(r)-\beta u_{\alpha \gamma}^{\mathrm{el}}(r)$, with $c_{\alpha \gamma}^{s}(r)$ denoting the short-range contributions. For ionic multicomponent systems, in which only a subset of the components can be detected experimentally, it is useful to define effective interactions between observable species, which are obtained by integrating out the degrees of freedom of the invisible species. Implementing the effective interactions into the DIT yields an effective electrostatic contribution of the general form $\beta \tilde{u}_{\alpha \gamma}^{e e}(q)=$ $\tilde{z}_{\alpha}^{(*)}(q) \tilde{v}^{(*)}(q) \tilde{z}_{\gamma}^{(*)}(q)$, where $x^{(*)}$ denotes renormalized values; $\tilde{v}^{(*)}(q)=4 \pi l_{b} /\left[q^{2}+\kappa_{B}^{2}(q)\right]$ and $\tilde{z}_{\alpha}^{(*)}(q)=\tilde{z}_{\alpha}(q)+\Delta \tilde{z}_{\alpha}(q)$ [14,24-26]. The effective screening function $\kappa_{B}^{2}(q)$ is entirely determined by the $\tilde{c}_{\alpha \gamma}^{s}(q)$ among the invisible species, and their densities, whereas the correction to the bare charge distribution, $\Delta \tilde{z}_{\alpha}(q)$, also involves the Fourier transform of the direct correlation functions between the subset of observable particles and the subset of invisible ones.

In our light-scattering experiments the species $a$ and $c$ belong to this last subset, while the observable subset is the species $M$. The asymptotic behavior of the effective interactions among microgels immersed in a simple electrolyte is determined by the poles of $\tilde{v}^{(*)}(q)=4 \pi l_{b} /\left[q^{2}+\kappa_{B}^{2}(q)\right]$ found in the upper half of the complex plane, those closest to the real axis. For standard conditions $q=i \eta$; this yields $v^{(*)}(r)=\left[l_{b} / E^{(*)}\right] \exp (-\eta r) / r$, where the effective permittivity $E^{(*)}$ comes from the residue of $\tilde{v}^{(*)}(q)[14]$ and $\eta$ is the renormalized screening parameter. Therefore, we obtain for the long-range component of the microgel-microgel effective potential

$$
\beta u_{M M}^{\mathrm{eff}}(r)=\frac{l_{B}}{E^{(*)} r} \exp (-\eta r) A_{M}^{2},
$$

with $A_{M} \equiv \tilde{z}_{M}^{(*)}(i \eta)$ being the renormalized charge of the microgel. If the magnitude of this renormalized charge is large enough, then this long-range component becomes dominant, such that the remaining contributions to the effective microgel-microgel interaction can be neglected.

A standard approximation would be to consider the small ions as pointlike, so that $\tilde{z}_{\alpha}(q)=Q_{\alpha}$, and to assume that the Debye-Hückel approximation applies, so that $c_{\alpha \gamma}^{s}(r)=0$. One then obtains $E^{(*)}=1$ and $\eta=\kappa_{\mathrm{se}}=\left[4 \pi l_{b}\left(\rho_{a} Q_{a}^{2}+\right.\right.$ $\left.\left.\rho_{c} Q_{c}^{2}\right)\right]^{1 / 2}$, with $\kappa_{\mathrm{se}}^{-1}$ being the Debye length of the supporting electrolyte. In the linear response approximation (LRA) first proposed by Denton [11], the term $\Delta \tilde{z}^{(*)}(q)$ is ignored, resulting in a renormalized charge,

$$
A_{M}^{(\mathrm{LRA})}(\eta)=\frac{F\left(\eta R_{M}\right) R_{M}^{3}-F\left(\eta S_{M}\right) S_{M}^{3}}{R_{M}^{3}-S_{M}^{3}} Q_{M},
$$

where for our microgel $F(x) \equiv 3[x \cosh (x)-\sinh (x)] / x^{3}$ [14].

However, accounting for the excluded volume of the microions and including the nonlinear contributions by implementing the hypernetted chain (HNC) approximation for the correlations among small ions and between microgels and surrounding clouds of microions, $\eta$ is not identical to $\kappa_{\mathrm{se}}$ and

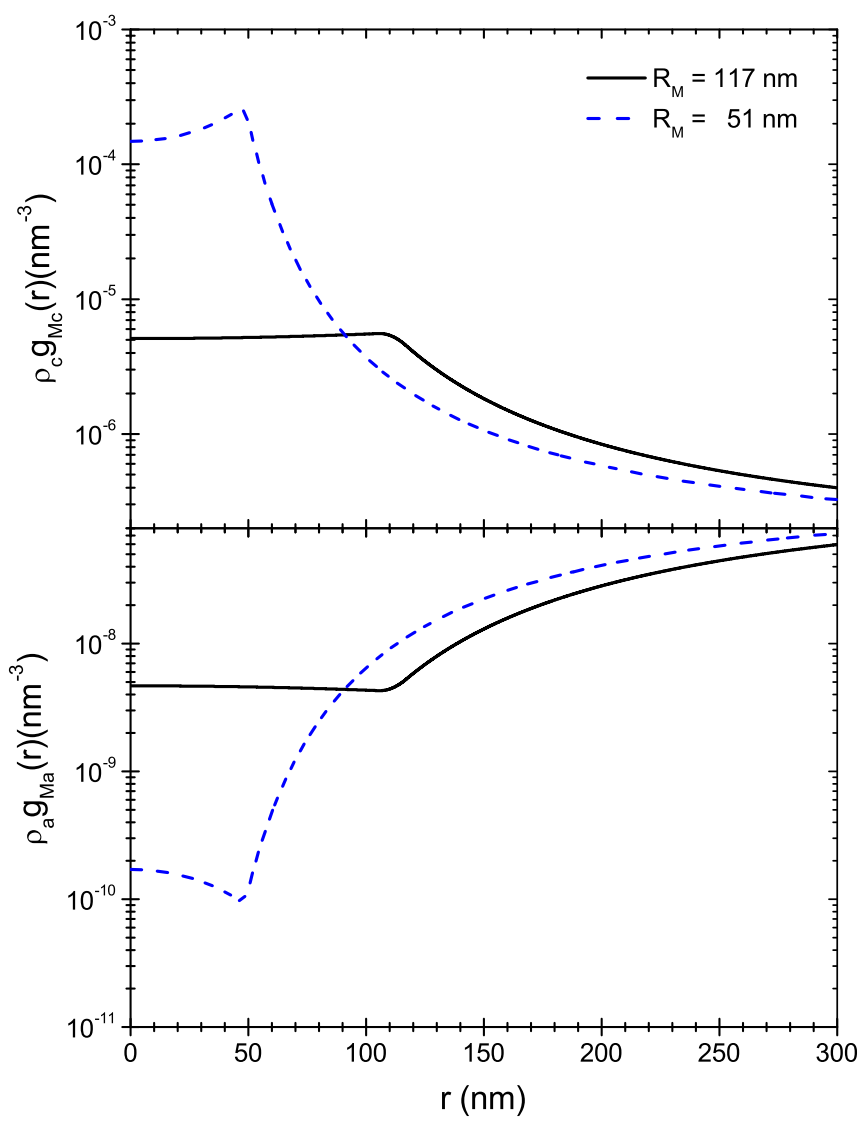

FIG. 1. Distribution profiles of counterions (top panel) and coions (bottom panel) around a model microgel with charge $Q_{M}=$ -720 in the infinite microgel dilution limit; $\theta_{s}=2.56 \times 10^{-7} \mathrm{M}$.

$E^{(*)} \neq 1$. We introduce a corresponding effective charge,

$$
Q_{M}^{\mathrm{eff}} \equiv \frac{R_{M}^{3}-S_{M}^{3}}{F\left(\eta R_{M}\right) R_{M}^{3}-F\left(\eta S_{M}\right) S_{M}^{3}} A_{M},
$$

that includes the nonlinear and excluded volume effects in the second term of $A_{M}=\tilde{z}_{M}(i \eta)+\Delta \tilde{z}_{M}(i \eta)$. Hence, this effective charge $Q_{M}^{\text {eff }}$ differs from the bare charge $Q_{M}$, as it comprises the effects from the short-ranged absorption of counterions and the short-ranged rejection of coions [14].

Nonetheless, let us in a first step consider the limit of infinite dilution of microgels $\left(\rho_{M}=0 \mathrm{~nm}^{-3}\right)$ for $\theta_{s}=2.56 \times$ $10^{-7} \mathrm{M}$. In our model $\rho_{a}=\theta_{s}$ with the conversion $1 \mathrm{M}=$ $0.60221413 \mathrm{~nm}^{-3}$. The corresponding Debye lengths is $\kappa_{\mathrm{se}}^{-1} \approx 600 \mathrm{~nm}$. Figure 1 shows the distribution profiles of counterions $\rho_{c} g_{\mathrm{Mc}}(r)$ (top panel) and coions $\rho_{a} g_{\mathrm{Ma}}(r)$ (bottom panel) for a bare charge of $Q_{M}=-720$ and $R_{M}=51 \mathrm{~nm}$ (dashed lines) and $R_{M}=117 \mathrm{~nm}$ (solid lines). Here $g_{\mathrm{Mc}}(r)$ and $g_{\mathrm{Ma}}(r)$ denote the radial distribution functions of counterions and coions, respectively. The most important thing to notice is that the number of counterions (small cations) inside the microgel sharply increases as $R_{M}$ decreases, whereas the number of coions (small anions) exhibits the opposite trend.

The boosted intake of counterions and the matching depletion of coions on microgel shrinking leads to a reduction in the magnitude of $Q_{M}^{\text {eff }}$ for large $\left|Q_{M}\right|$, as shown in Fig. 2. For 


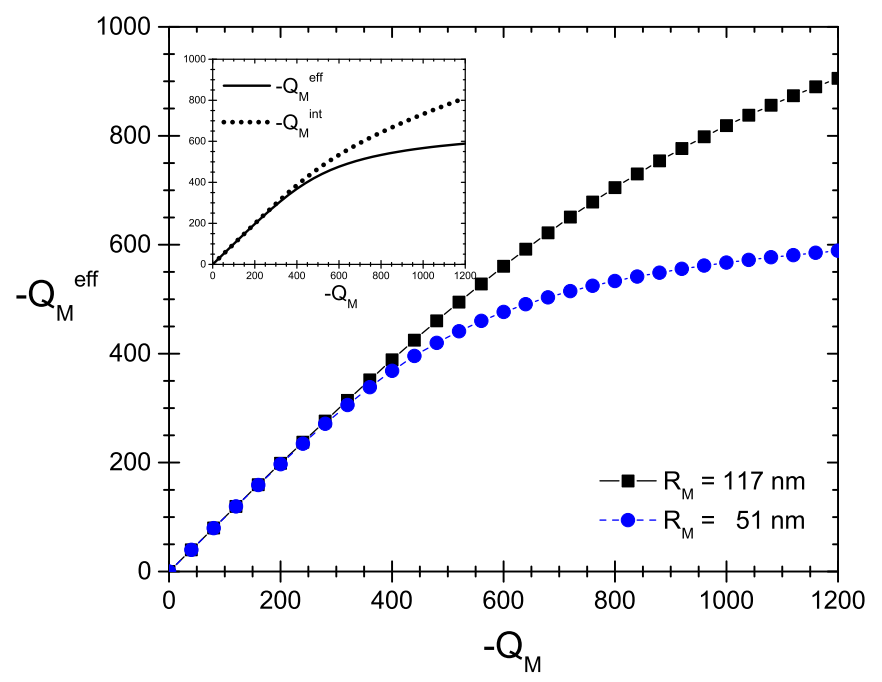

FIG. 2. Effective charge as a function of the bare charge in the limit of infinite microgel dilution; $\theta_{s}=2.56 \times 10^{-7} \mathrm{M}$. The inset illustrates the discrepancy between $Q_{M}^{\text {int }}$ and $Q_{M}^{\text {eff }}$ for the case of $R_{M}=51 \mathrm{~nm}$ and $\theta_{s}=2.56 \times 10^{-7} \mathrm{M}$, where $Q_{M}^{\text {int }} \equiv Q_{M}+$ $4 \pi \int_{0}^{R_{M}} r^{2} d r\left[g_{\mathrm{Ma}}(r) \rho_{a} q_{a}+g_{\mathrm{Mc}}(r) \rho_{c} q_{c}\right][12,13]$.

small values of $\left|Q_{M}\right|$, the relation between $\left|Q_{M}\right|$ and $\left|Q_{M}^{\text {eff }}\right|$ is essentially linear, but $Q_{M}^{\text {eff }}$ becomes almost constant at larger $\left|Q_{M}\right|$. The onset to saturation depends on the magnitude of $R_{M}$; the smaller the microgel size the earlier the onset to saturation, which effectively entails a decrease of $\left|Q_{M}^{\text {eff }}\right|$ with decreasing size provided that $\left|Q_{M}\right|$ is large enough.

Though these nonlinear effects are related to the features of the distribution profiles shown in Fig. 1, the values of $Q_{M}^{\text {eff }}$ are effectively determined by the profiles derived from the relevant short-range part of the direct correlation function $c_{\alpha \gamma}^{s}(r)$ [14,24-26]. This results in an effective charge that is distinct from the internal net charge $Q_{M}^{\text {int }}$ obtained from the simple addition of the charge of coions and counterions located inside the microgel and the bare charge $Q_{M}[12,13]$. This is illustrated in the inset of Fig. 2, where we show $Q_{M}^{\text {eff }}$ and $Q_{M}^{\text {int }}$ as a function of $Q_{M}$ for $R_{M}=51 \mathrm{~nm}$ and $\theta_{s}=$ $2.56 \times 10^{-7} \mathrm{M}$.
To extend our considerations to systems with finite concentration we propose the following approach. First, we implement a least-squares fitting for the quantities $Q_{M}^{\text {eff }}\left(\kappa_{\mathrm{se}}\right)$, $E^{(*)}\left(\kappa_{\mathrm{se}}\right)$, and $\eta\left(\kappa_{\mathrm{se}}\right)$ obtained for $\rho_{M}=0$ with six different concentrations of supporting electrolyte: $\theta_{s}=2.56 \times 10^{-7} \mathrm{M}$, $4 \times 10^{-7} \mathrm{M}, 16 \times 10^{-7} \mathrm{M}, 64 \times 10^{-7} \mathrm{M}, 256 \times 10^{-7} \mathrm{M}$, and $1024 \times 10^{-7} \mathrm{M}$. These concentrations are unrelated to the experimental conditions considered in Sec. II, they are used here just to analyze the dependence of $Q_{M}^{\text {eff }}$ on the ionic strength of the supporting electrolyte and correspond, respectively, to the Debye lengths: $\kappa_{\mathrm{se}}^{-1} \approx 600,480,240,120$, 60 , and $30 \mathrm{~nm}$. Taking into account that $Q_{M}^{\mathrm{eff}} \rightarrow Q_{M}, E^{(*)} \rightarrow$ 1 , and $\eta / \kappa_{\text {se }} \rightarrow 1$ when $\kappa_{\text {se }} \rightarrow 0$, we employ the following functional forms:

$$
\begin{aligned}
& Q_{M}^{\mathrm{eff}}\left(\kappa_{\mathrm{se}}\right)=Q_{M}+A \kappa_{\mathrm{se}}^{\nu} \exp \left(-C \kappa_{\mathrm{se}}^{1 / 2}\right), \\
& l_{R}\left(\kappa_{\mathrm{se}}\right)=l_{b}+\left(a_{0}+a_{1} \kappa_{\mathrm{se}}+a_{2} \kappa_{\mathrm{se}}^{2}\right) \kappa_{\mathrm{se}}
\end{aligned}
$$

and

$$
\eta\left(\kappa_{\mathrm{se}}\right)=\kappa_{\mathrm{se}}+\left(c_{0}+c_{1} \kappa_{\mathrm{se}}+c_{2} \kappa_{\mathrm{se}}^{2}\right) \kappa_{\mathrm{se}}^{2},
$$

where the adjustable parameters $A, v$, and $C$ depend on $R_{M}$ and $Q_{M}$, whereas $a_{0}, a_{1}, a_{2}, c_{0}, c_{1}$, and $c_{2}$ are independent of $R_{M}$ and $Q_{M}$.

For $\rho_{M}>0$ we then distinguish between counterions that are bound to the microgel and those that are free to move throughout the solution, such that $\rho_{c}=\rho_{c}^{b}+\rho_{c}^{f}$. We further assume that the bound (condensed) counterions are responsible for the difference between effective charge and bare charge; that is, $Q_{M}^{\text {eff }}=Q_{M}+Q_{c} \rho_{c}^{b} / \rho_{M}$. The electroneutrality condition

$$
\rho_{M} Q_{M}^{\mathrm{eff}}+\rho_{a} Q_{a}+\rho_{c}^{f} Q_{c}=0
$$

then provides the connection between the effective charge and the density of freely moving counterions at finite microgel concentration. Using also the relation $\kappa_{\mathrm{se}}=\left[4 \pi l_{b}\left(\rho_{a} Q_{a}^{2}+\right.\right.$ $\left.\left.\rho_{c}^{f} Q_{c}^{2}\right)\right]^{1 / 2}$, we obtain the values of $\kappa_{\mathrm{se}}$ at varying $\rho_{M}$ and $Q_{M}^{\text {eff }}$; this relationship can be inverted to provide $Q_{M}^{\text {eff }}\left(\kappa_{\mathrm{se}}\right)$ for $\rho_{M}>0$. The intersection of this last function with the function $Q_{M}^{\text {eff }}\left(\kappa_{\mathrm{se}}\right)$ given in (4), valid for $\rho_{M}=0$, yields the values of $\kappa_{\mathrm{se}}$

\begin{tabular}{|c|c|c|c|c|c|c|c|c|}
\hline & $R_{M} / \mathrm{nm}$ & $\rho_{M} / \mathrm{nm}^{-3}$ & $\phi$ & $\theta_{s} / \mathrm{M}$ & $Q_{M}^{\mathrm{eff}}$ & $A_{M}$ & $\eta^{-1} / \mathrm{nm}$ & $E^{(*)}$ \\
\hline Fig. 3(a) & 117 & $1.3 \times 10^{-9}$ & 0.0087 & $10^{-7}$ & -626 & -637.21 & 345.24 & 1.00000015 \\
\hline Fig. 3(b) & 89 & $1.3 \times 10^{-9}$ & 0.0038 & $10^{-7}$ & -587 & -592.83 & 355.02 & 1.00000014 \\
\hline Fig. 3(c) & 55 & $1.3 \times 10^{-9}$ & 0.0009 & $10^{-7}$ & -495 & -496.75 & 381.80 & 1.00000013 \\
\hline Fig. 3(d) & 51 & $1.3 \times 10^{-9}$ & 0.0007 & $10^{-7}$ & -479 & -479.93 & 387.28 & 1.00000012 \\
\hline Fig. 4(a) & 117 & $6.9 \times 10^{-9}$ & 0.0463 & $10^{-7}$ & -618 & -670.06 & 159.34 & 1.00000071 \\
\hline Fig. 4(b) & 89 & $6.9 \times 10^{-9}$ & 0.0204 & $10^{-7}$ & -563 & -587.28 & 166.81 & 1.00000065 \\
\hline Fig. 4(c) & 55 & $6.9 \times 10^{-9}$ & 0.0048 & $10^{-7}$ & -451 & -456.68 & 185.71 & 1.00000052 \\
\hline Fig. 4(d) & 51 & $6.9 \times 10^{-9}$ & 0.0038 & $10^{-7}$ & -433 & -437.79 & 189.31 & 1.00000050 \\
\hline Fig. 5(a) & 117 & $6.9 \times 10^{-9}$ & 0.0463 & $10^{-7}$ & -618 & -670.06 & 159.34 & 1.00000071 \\
\hline Fig. 5(b) & 117 & $6.9 \times 10^{-9}$ & 0.0463 & $3 \times 10^{-6}$ & -628 & -725.27 & 118.38 & 1.00000128 \\
\hline Fig. 5(c) & 117 & $6.9 \times 10^{-9}$ & 0.0463 & $4 \times 10^{-6}$ & -632 & -745.07 & 110.18 & 1.00000148 \\
\hline Fig. 5(d) & 117 & $6.9 \times 10^{-9}$ & 0.0463 & $1.3 \times 10^{-5}$ & -656 & -930.51 & 74.29 & 1.00000324 \\
\hline
\end{tabular}
and $Q_{M}^{\text {eff }}$ (i.e., $A_{M}$ ) used to fit the experimental results for given values of $\rho_{M}$ and $R_{M}$, where the remaining fitting parameter

TABLE I. Values used in the corresponding one component model for $Q_{M}=-720$. 
is thus the bare charge $Q_{M}$. For the sake of consistency, we assume the values of $l_{R}$ and $\eta$ corresponding to such value of $\kappa_{\mathrm{se}}$, though their respective deviations from $l_{b}$ and $\kappa_{\mathrm{se}}$ are basically negligible.

\section{THEORY VERSUS EXPERIMENT}

For stringent testing of our theory, we explore structural correlations among PNiPAM microgels using static lightscattering experiments, as described in Sec. II. We produce three data sets. In the two first sets we use two microgel systems differing in microgel concentration and determine $S(q)$ as a function of the microgel size, which we vary by changing the temperature. The two microgel systems are kept at quasideionized conditions, where we assume that $\theta_{s}=10^{-7} \mathrm{M}$. In the third set, we fix the microgel size and concentration and determine $S(q)$ as a function of added salt.

The structure factors obtained for the two systems at quasideionized conditions, with microgel concentrations of, respectively, $\rho_{M}=1.3 \times 10^{-9} \mathrm{~nm}^{-3}$ and $\rho_{M}=6.9 \times$ $10^{-9} \mathrm{~nm}^{-3}$, are shown in Figs. 3 and 4. For $T=24.7^{\circ} \mathrm{C}$, these number concentrations correspond to particle volume fractions of $\phi=0.009$ and $\phi=0.046$, as shown in Table I. Despite of these rather low volume fractions the microgels adopt strongly correlated configurations. This is evidenced by the high magnitude of the first peak in the liquid structure factor of the system with $\rho_{M}=1.3 \times 10^{-9} \mathrm{~nm}^{-3}$ and by the presence of Bragg peaks in $S(q)$ for the system with $\rho_{M}=6.9 \times 10^{-9} \mathrm{~nm}^{-3}$. For both systems the degree of correlation decreases on increasing the temperature, i.e., decreasing the particle size. This is evidenced by the decrease in the magnitude of the first peak in $S(q)$ for the system with $\rho_{M}=1.3 \times 10^{-9} \mathrm{~nm}^{-3}$ and even more spectacularly by the melting of the crystalline configuration of the system with $\rho_{M}=6.9 \times 10^{-9} \mathrm{~nm}^{-3}$. In the latter case, $S(q)$ exhibits Bragg peaks typical of crystalline states at low temperatures, while exhibiting the features of typical fluid states at higher temperatures. The position of the first peak remains in both systems unchanged on changing the particle size. This shows that the electrostatic repulsion among the microgels is sufficiently strong to maintain neighboring particles at maximal distance. However, the decrease in the degree of correlation of the particle position with increasing temperature indicates that the effective interaction among the microgels decreases markedly with decreasing particle size, as already denoted in a previous work [4].

Increasing the ionic strength for the system with $\rho_{M}=$ $6.9 \times 10^{-9} \mathrm{~nm}^{-3}$ has a similar effect on the degree of correlation as decreasing the particle size. On increasing $\theta_{s}$ at a fixed particle size $\left(T=24.7^{\circ} \mathrm{C}\right)$ leads to melting of the crystalline configuration obtained at quasideionized conditions. This is shown in Fig. 5, displaying from top to bottom the structure factor obtained for the system at quasideionized conditions, in contact with air and no added salt and with $10^{-6} \mathrm{M}$ and $10^{-5} \mathrm{M}$ of added $\mathrm{NaCl}$. Again the position of the first peak is basically fixed, but the increased screening of the macroion charge leads to a decrease of correlation among microgels.

To approximate the experimental data we use the HNC approximation for a one component fluid with pair interactions described by Eq. (1), and we follow the procedure

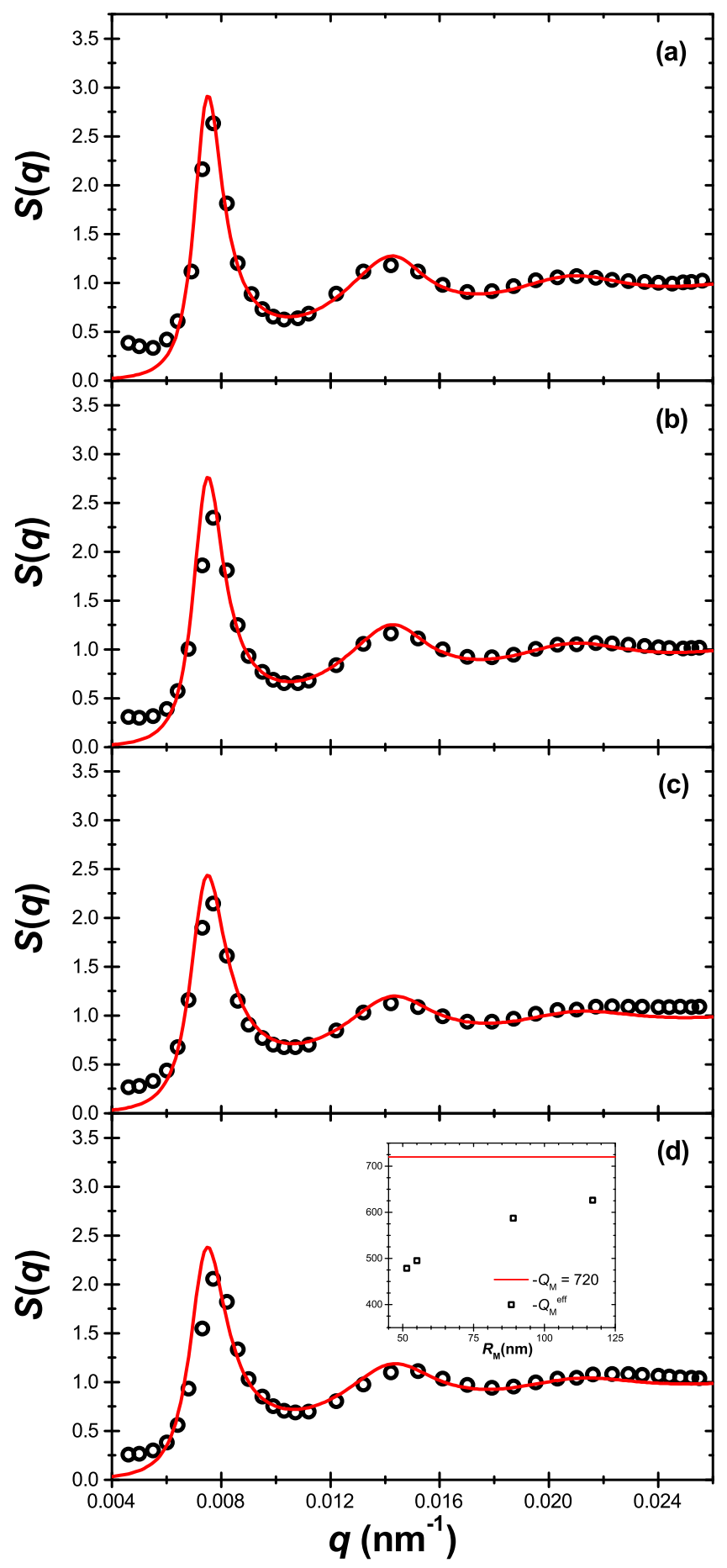

FIG. 3. Structure factors for $\rho_{M}=1.3 \times 10^{-9} \mathrm{~nm}^{-3}$ and $\rho_{a}=$ $0.60221413 \times 10^{-7} \mathrm{~nm}^{-3}$ : (a) $T=24.7^{\circ} \mathrm{C}, R_{M}=117 \mathrm{~nm}, \phi=$ 0.00872 ; (b) $T=31.1^{\circ} \mathrm{C}, R_{M}=89 \mathrm{~nm}, \phi=0.00384$; (c) $T=$ $33.2^{\circ} \mathrm{C}, R_{M}=55 \mathrm{~nm}, \phi=0.00091$; and (d) $T=34.9^{\circ} \mathrm{C}, R_{M}=$ $51 \mathrm{~nm}, \phi=0.00072$. The circles represent the experimental data and the solid lines correspond to theory with $Q_{M}=-720$. Inset in (d): Effective charge as a function of the microgel radius.

described in Sec. III to obtain $A_{M}, \eta$, and $l_{R}$. For all data sets obtained with fluid systems, a good agreement between theory and experiment is obtained by assuming a bare charge of $Q_{M}=-720$; the corresponding theoretical results are shown 


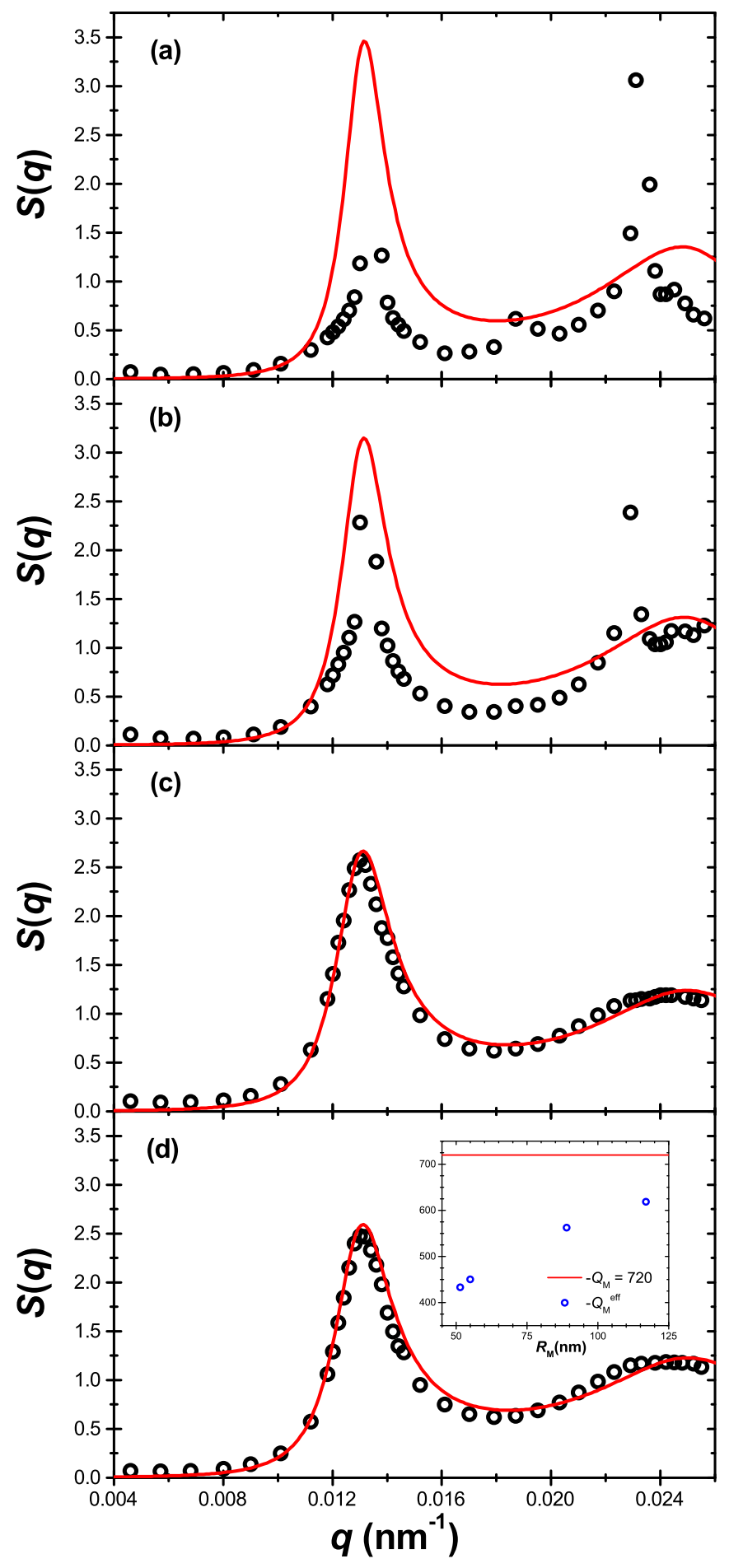

FIG. 4. Structure factors for $\rho_{M}=6.9 \times 10^{-9} \mathrm{~nm}^{-3}$ and $\rho_{a}=$ $0.60221413 \times 10^{-7} \mathrm{~nm}^{-3}$ : (a) $T=24.7^{\circ} \mathrm{C}, R_{M}=117 \mathrm{~nm}, \phi=$ 0.0463 ; (b) $T=31.1^{\circ} \mathrm{C}, R_{M}=89 \mathrm{~nm}, \phi=0.0204$; (c) $T=33.2^{\circ} \mathrm{C}$, $R_{M}=55 \mathrm{~nm}, \phi=0.0048$; and (d) $T=34.9^{\circ} \mathrm{C}, R_{M}=51 \mathrm{~nm}, \phi=$ 0.0038 . For clarity we omitted in (a) and (b) the data obtained at the Bragg peaks, where $S(q)$ significantly exceeds 3.5 . The circles represent the experimental data and the solid lines correspond to theory with $Q_{M}=-720$. Inset in (d): Effective charge as a function of the microgel radius.

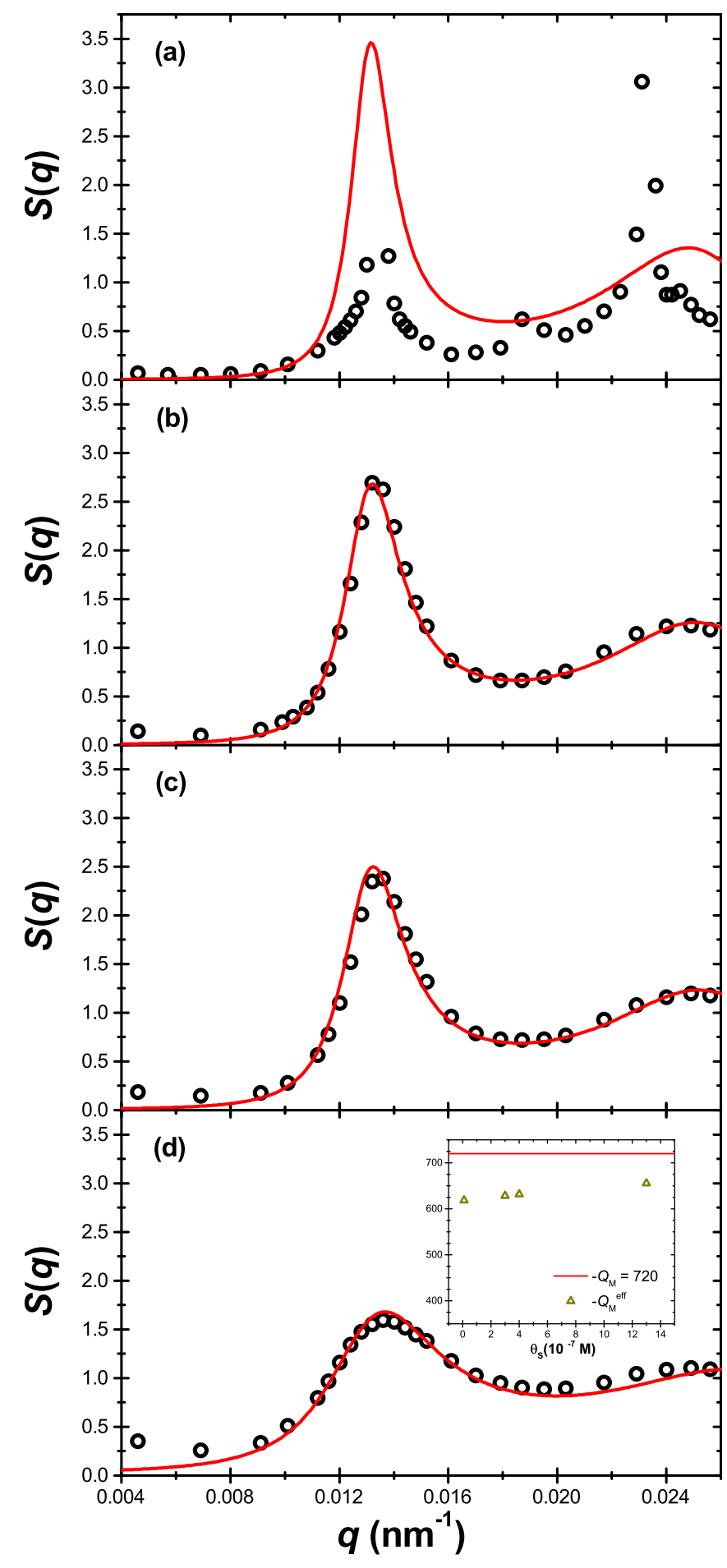

FIG. 5. Structure factors for $\rho_{M}=6.9 \times 10^{-9} \mathrm{~nm}^{-3}$, $R_{M}=117 \mathrm{~nm}$ and $\phi=0.046$ (measured at $T=24.7^{\circ} \mathrm{C}$ ): (a) $\rho_{a}=6.0221413 \times 10^{-8} \mathrm{~nm}^{-3}$, (b) $\rho_{a}=1.80664239 \times 10^{-6} \mathrm{~nm}^{-3}$, (c) $\rho_{a}=2.40885652 \times 10^{-6} \mathrm{~nm}^{-3}$, and (d) $\rho_{a}=7.82878369 \times$ $10^{-6} \mathrm{~nm}^{-3}$. For clarity we omitted in (a) the data obtained at the Bragg peaks, where $S(q)$ significantly exceeds 3.5 . The circles represent the experimental data and the solid lines correspond to theory with $Q_{M}=-720$. Inset in (d): Effective charge as a function of the salt concentration. 
as solid lines in Figs. 3 to 5. Moreover, we find that the magnitude of the first peak of the theoretical structure factor exceeds the Hansen-Verlet criterion for freezing, $S\left(q_{\max }\right)>$ $2.85[27,28]$, for all systems exhibiting a crystalline structure, which corroborates that our theory properly accounts for the effective interactions among our microgels.

Based on the evaluations of the charge distribution at infinite microgel dilution, we expect that $Q_{M}^{\text {eff }}$ will depend on the microgel size for $Q_{M}=-720$ (see Figs. 1 and 2). At the finite microgel concentrations investigated here this is the case as well, as shown in the insets of Fig. 3(d) and Fig. 4(d) and in Table I. However, let us also note that $Q_{M}^{\text {eff }}$ decreases on increasing the microgel concentration. This can be understood as being due to the increase of the average counterion concentration, which favors condensation. By contrast, increasing the average ion concentration, including coions and counterions, by increasing the salt concentration may favor the release of condensed ions, as the average electrostatic potential in the bulk becomes comparable to that within the microgel volume, and a similar effect is observed in plain colloids [29]. Consequently, $\left|Q_{M}^{\text {eff }}\right|$ increases with increasing salt concentration, as shown in the inset of Fig. 5(d).

\section{CONCLUDING REMARKS}

In this paper, we show that effective interactions among permeable charged spheres, such as microgels, can be described by theories that include nonlinear effects, such as counterion condensation. The strength of this theory is its ability to account for structural correlations of charged microgel systems at different conditions with a unique value of the microgel bare charge. Attempting to describe these structural correlations by using linear response approximations [see Eq. (2)] requires adapting the bare charge ad hoc. Our model may be further improved by considering the dissimilarities among the different species of small ions, as well as excluded volume effects due to the presence of polymer segments. More importantly, the real effects of a finite macroion concentration may be studied employing a more robust approach for the theoretical determination of the correlations among all the ionic components.

\section{ACKNOWLEDGMENTS}

The authors thank the General Coordination of Information and Communications Technologies (CGSTIC) at Cinvestav for providing the HPC resources of the Hybrid Cluster Supercomputer "Xiuhcoatl" (LANCAD CINVESTAV node) that greatly contributed to the research results reported in this paper. We also gratefully acknowledge support from Conacyt through Grant No. 397911 and Project No. A1-S-16002 and from the Swiss National Science Foundation (Project No. 200020-138212). J.C.-F. thanks the financial support provided by the Spanish Ministerio de Economía y Competitividad, Plan Nacional de Investigación, Desarrollo e Innovación Tecnológica (Project No. FIS2016-80087-C2-1-P) and the European Regional Development Fund.
[1] W. B. Russel, D. A. Saville, and W. R. Schowalter, Colloidal Dispersions (Cambridge University Press, Cambridge, 1989).

[2] E. J. W. Verwey and J. Th. G. Overbeek, Theory of the Stability of Lyophobic Colloids (Elsevier, New York, 1948).

[3] M. Ledesma-Motolinía, M. Braibanti, L. F. Rojas-Ochoa, and C. Haro-Pérez, Coll. Surf. A 482, 724 (2015).

[4] M. Braibanti, C. Haro-Pérez, M. Quesada-Pérez, L. F. Rojas-Ochoa, and V. Trappe, Phys. Rev. E 94, 032601 (2016).

[5] H. Senff and W. Richtering, J. Chem. Phys. 111, 1705 (1999).

[6] M. Bradley, J. Ramos, and B. Vincent, Langmuir 21, 1209 (2005).

[7] P. Holmqvist, P. S. Mohanty, G. Nägele, P. Schurtenberger, and M. Heinen, Phys. Rev. Lett. 109, 048302 (2012).

[8] G. Aguirre, J. Ramos, and J. Forcada, Soft Matter 9, 261 (2013).

[9] V. Nigro, R. Angelini, M. Bertoldo, V. Castelvetro, G. Ruocco, and B. Ruzicka, J. Non-Cryst. Solids 407, 361 (2015).

[10] J. Maldonado-Valderrama, T. del Castillo-Santaella, I. AdroherBenítez, A. Moncho-Jordá, and A. Martín-Molina, Soft Matter 13, 230 (2017).

[11] A. R. Denton, Phys. Rev. E 67, 011804 (2003); 68, 049904 (2003).

[12] A. Moncho-Jordá, J. A. Anta, and J. Callejas-Fernández, J. Chem. Phys. 138, 134902 (2013).
[13] A. Moncho-Jordá and I. Adroher-Benítez, Soft Matter 10, 5810 (2014).

[14] P. González-Mozuelos, J. Chem. Phys. 144, 054902 (2016).

[15] M. Quesada-Pérez, J. A. Maroto-Centeno, A. MartínMolina, and A. Moncho-Jordá, Phys. Rev. E 97, 042608 (2018).

[16] R. Kjellander and D. J. Mitchell, Molecular Phys. 91, 173 (1997).

[17] P. S. Mohanty, S. Nöjd, M. J. Bergman, G. Nägele, S. ArreseIgor, A. Alegria, R. Roa, P. Schurtenberger, and J. K. G. Dhont, Soft Matter 12, 9705 (2016).

[18] J. Zhou, J. Wei, T. Ngai, L. Wang, D. Zhu, and J. Shen, Macromolecules 45, 6158 (2012).

[19] M. Stieger, W. Richtering, J. S. Pedersen, and P. Lindner, J. Chem. Phys. 120, 6197 (2004).

[20] C. Urban and P. Schurtenberger, J. Colloid Interface Sci. 207, 150 (1998).

[21] C. Haro-Pérez, G. J. Ojeda-Mendoza, and L. F. Rojas-Ochoa, J. Chem. Phys. 134, 244902 (2011)

[22] G. J. Ojeda-Mendoza, A. Moncho-Jordá, P. GonzálezMozuelos, C. Haro-Pérez, and L. F. Rojas-Ochoa, Soft Matter 14, 1355 (2018).

[23] P. González-Mozuelos, J. Phys. Chem. B 110, 22702 (2006).

[24] P. González-Mozuelos and M. D. Carbajal-Tinoco, J. Chem. Phys. 109, 11074 (1998). 
[25] M. D. Carbajal-Tinoco and P. González-Mozuelos, J. Chem. Phys. 117, 2344 (2002).

[26] P. González-Mozuelos, G. I. Guerrero-García, and M. Olvera de la Cruz, J. Chem. Phys. 139, 064709 (2013).

[27] J. P. Hansen and L. Verlet, Phys. Rev. 184, 151 (1969).
[28] H. Löwen, T. Palberg, and R. Simon, Phys. Rev. Lett. 70, 1557 (1993).

[29] L. F. Rojas-Ochoa, R. Castaneda-Priego, V. Lobaskin, A. Stradner, F. Scheffold, and P. Schurtenberger, Phys. Rev. Lett. 100, 178304 (2008). 